\title{
INTEGRACIÓN EMPRESARIAL, UNA POSICIÓN ESTRATÉGICA
}

\section{Abilio Tinoco León}

Gerente general de BPI Center SAC

\section{Resumen}

La integración empresarial necesita el alineamiento de estrategias, objetivos, procesos, sistemas y la infraestructura de tecnologías de información, así como la coordinación de las actividades interfuncionales que hacen uso de la tecnología de información. El alineamiento y coordinación requieren un conjunto de métodos de diagramación para representar los diferentes aspectos del negocio para cumplir ciertos propósitos. Las arquitecturas empresariales proveen de una serie de estos métodos que permiten la realización de un modelo empresarial integrado. Dicha integración — mediante una arquitectura empresarial — provee la base para facilitar la implementación de las iniciativas estratégicas.

Palabras clave

Arquitectura empresarial, coreografía, mapa estratégico, mapa de procesos, modelo de operación, orquestación, proceso de negocio, servicio y SOA. 


\section{Introducción}

En la actualidad, los clientes son más exigentes en lo que se refiere a precios, servicio y calidad. Esto obliga a las empresas a conocer mejor los requerimientos de los usuarios, reducir el tiempo de mercadeo, bajar los costos de producción y mejorar la calidad de sus productos; del mismo modo, tienen que cumplir con las regulaciones. ¿Cómo enfrentar estos desafíos?

\section{Mapa de procesos}

Una organización es tan buena como lo son sus procesos (Rummbler y Brache). Entonces, ¿en cuál de los procesos y en qué debemos ser buenos (figura 1).
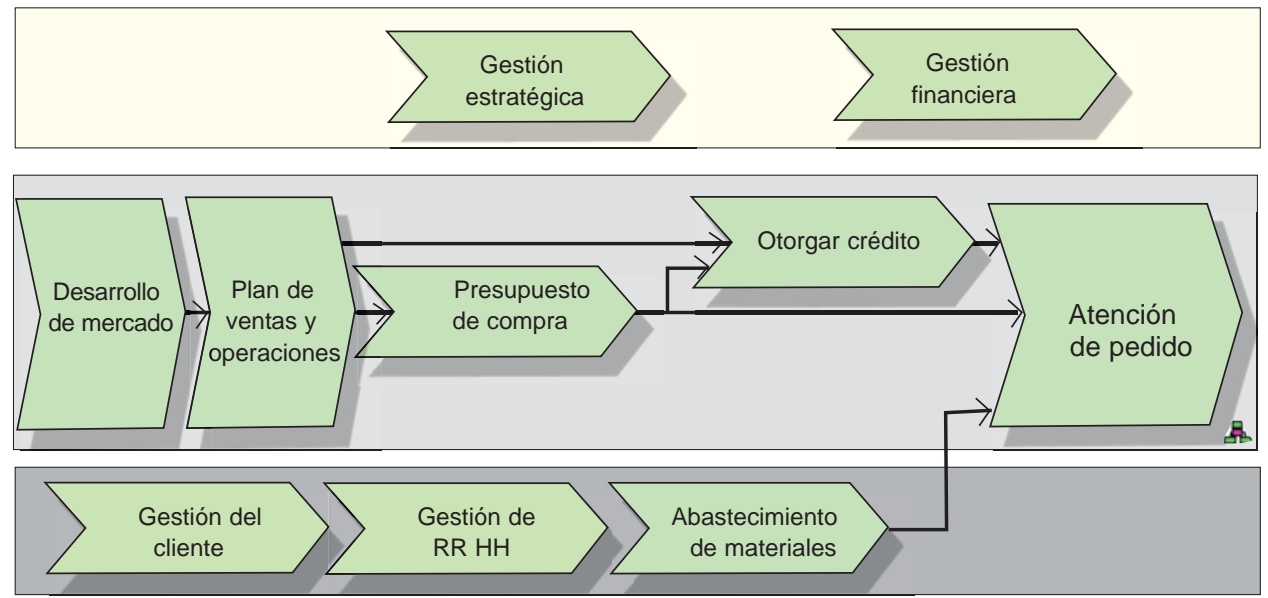

Figura 1. Mapa de procesos.

\section{Posición estratégica}

El éxito proviene de explotar una posición estratégica singular. Una posición estratégica no es otra cosa que la suma de las respuestas que una empresa da a las siguientes preguntas: ¿Quiénes deben ser mis clientes? ¿Qué productos o servicios les debo ofrecer? ¿Cómo debo hacerlo? (Constantinos C. Markides). Esto es, alinear el segmento de clientes, la propuesta de valor y los procesos. 


\section{El cuadro de mando integral}

Los esfuerzos tienen mejores resultados cuando están bien dirigidos, lo cual implica conocer las estrategias y los objetivos estratégicos del negocio. Sobre todo, es importante saber cuáles son los objetivos de los procesos, cuál es la propuesta de valor que deben cumplir los procesos (por ejemplo, mayor facilidad de compra); o cuáles son los resultados financieros esperados por los procesos (por ejemplo, reducir costos) (figura 2).

Los objetivos de los procesos influyen para el cumplimiento de la propuesta de valor al cliente $y$, como consecuencia, alcanzar los objetivos financieros; o también directamente para alcanzar los objetivos mencionados (figura 2). Entonces, la mejoría de los procesos puede orientarse a añadir valor para el cliente o a mejorar la eficiencia del proceso.

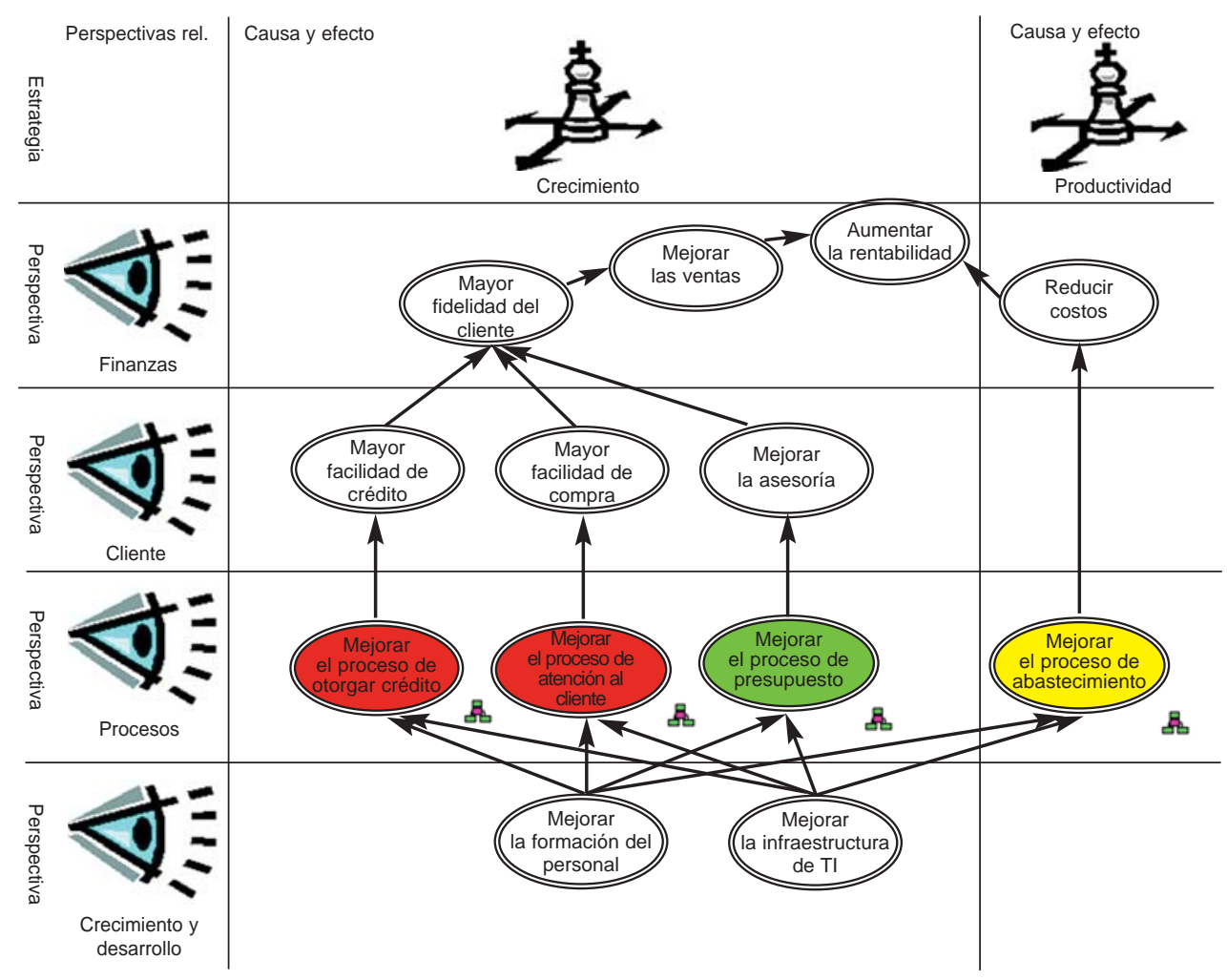

Figura 2. Mapa estratégico 
Para alcanzar los objetivos de los procesos se necesita poner en marcha; por ejemplo, implementar eCommerce (figura 3). Estas iniciativas muchas veces resultan ser proyectos de TI (tecnología de la información). Asimismo, se requiere el apoyo de los procesos, sin estos no hay forma de alcanzar los objetivos. Entonces, debemos alinear los procesos a sus objetivos (figura 4).

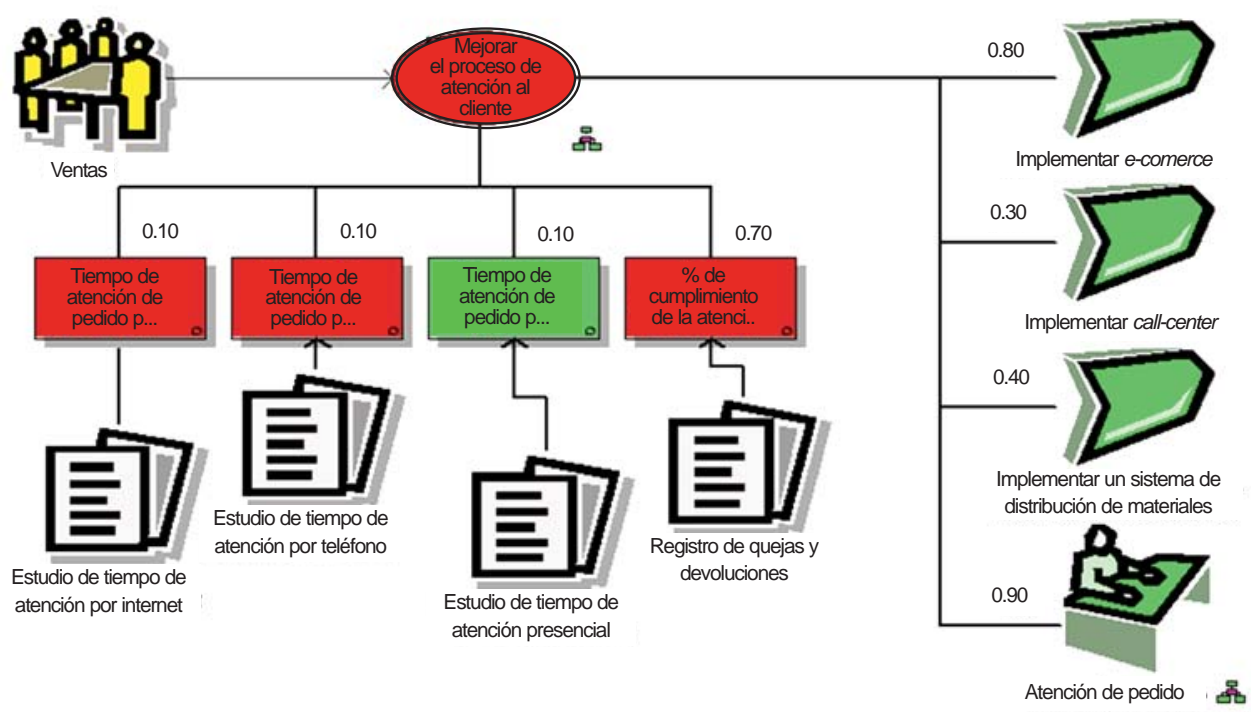

Figura 3. Diagrama de números indicadores.

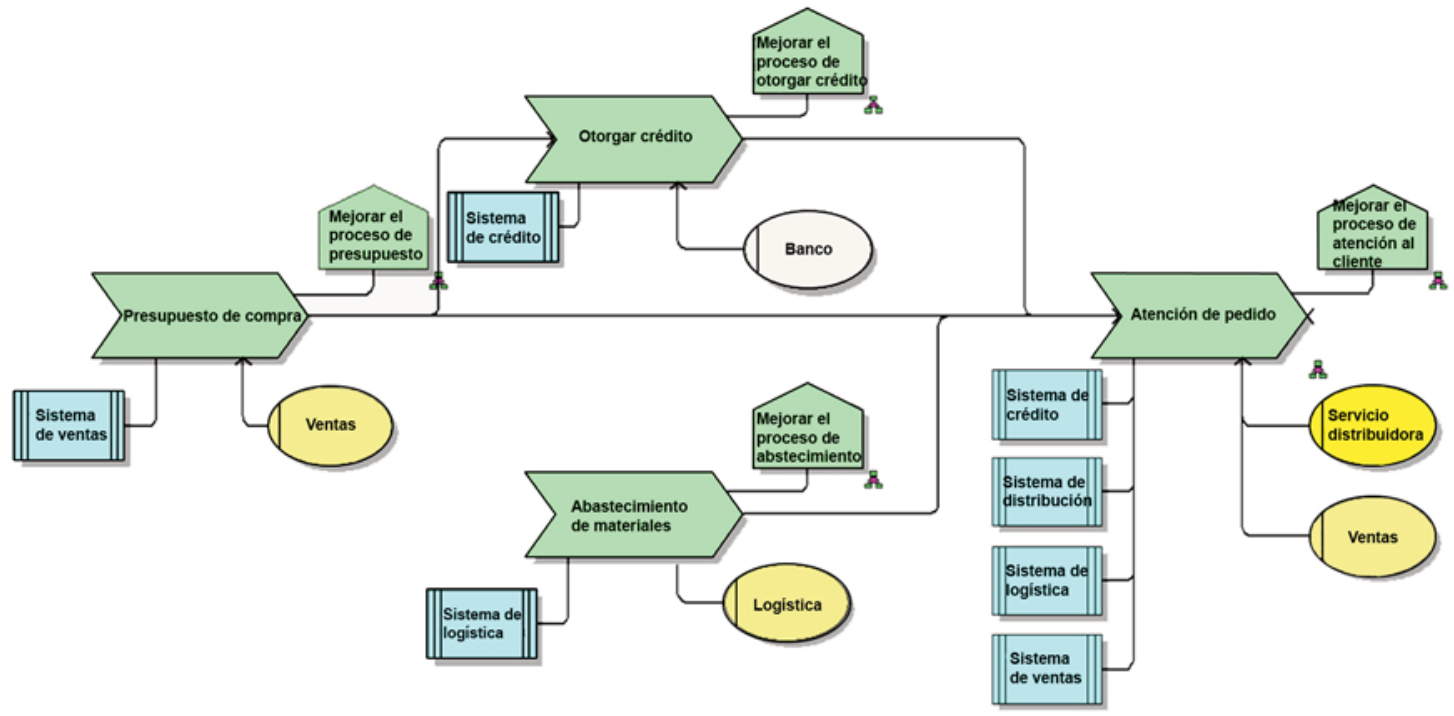

Figura 4. Red de valor 
La red de valor es la fuente de la ventaja competitiva que provee recursos: software de aplicaciones para manejar las políticas comerciales, out-tasking para realizar actividades especializadas e in-sourcing para mejorar la relación con el cliente (Keen y MacDonald). Para esto se requiere la colaboración de otras entidades, como los bancos, por ejemplo (figura 4).

\section{Bases para la ejecución de una estrategia}

Las bases para la ejecución de una estrategia resultarán de una selección cuidadosa de procesos y sistemas a ser estandarizados e integrados. Requiere de tres patrones claves: modelo de operación, arquitectura empresarial y modelo de ajuste TI.

El modelo operacional es la base para la implementación rápida de las iniciativas estratégicas con el fin de brindar buenos servicios a los clientes (Ross, Weill y Robertson). Un modelo operacional tiene dos dimensiones: integración y estandarización.

La integración coordina los esfuerzos compartiendo datos, por ejemplo, el crédito (figura 5). Los beneficios de la integración incluyen mejora de la eficiencia, coordinación, transparencia y agilidad. Un conjunto de procesos de negocios integrados puede mejorar los servicios al cliente y permite predecir los cambios de alguna parte y alertar a otras. La integración puede aumentar la velocidad del flujo de información y de las transacciones interfuncionales.

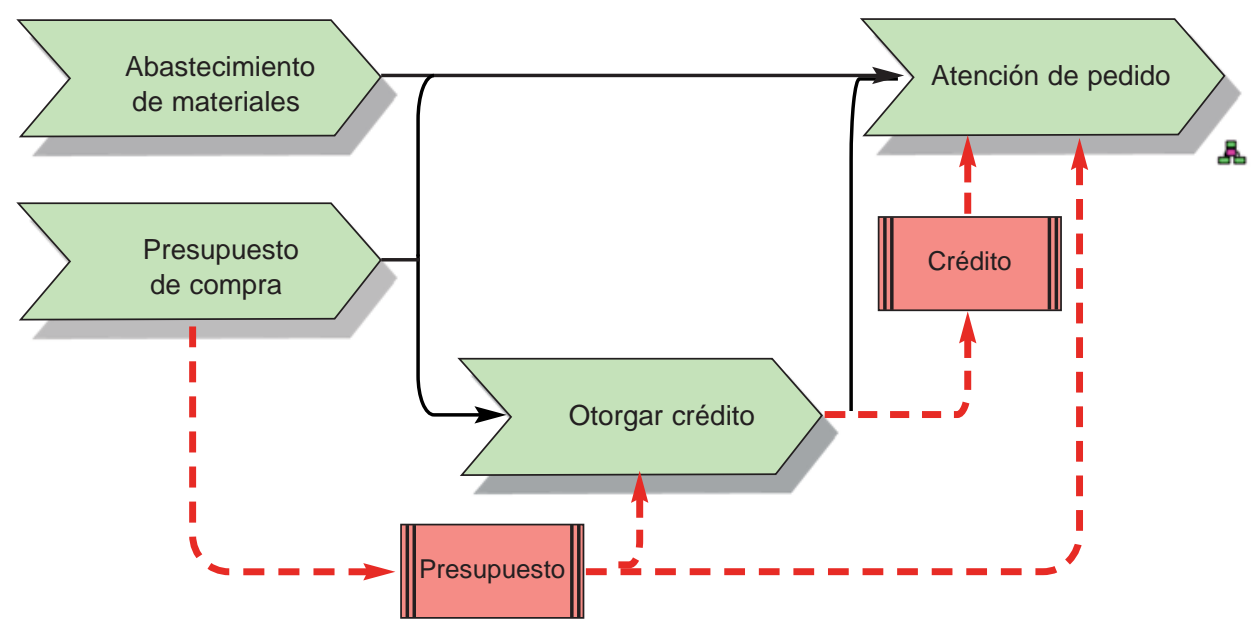

Figura 5. La integración de procesos. 
La estandarización define la forma como será ejecutado el proceso de negocio; es decir, la orquestación y la coreografía. La estandarización provee eficiencia y predicción interfuncional e interempresarial. Puede mejorar dramáticamente la eficiencia.

Para definir la estandarización es indispensable conocer la visión del proceso (figura 6), el cual se define con los objetivos y características del proceso (Davenport).

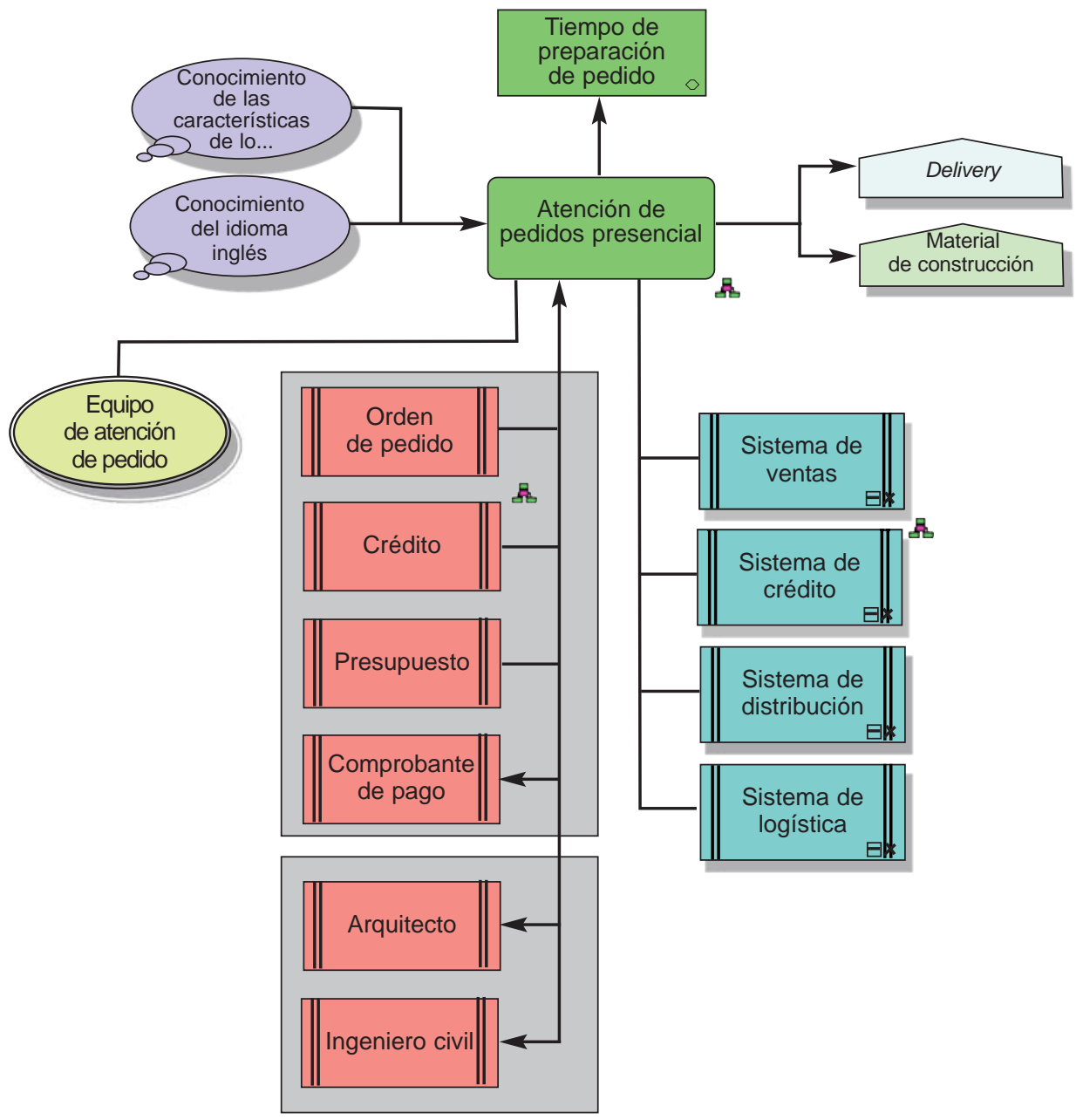

Figura 6. Visión del proceso. 
Los procesos deben ser flexibles y rápidos en adaptarse a los requerimientos del cliente; la integración de los sistemas para compartir los datos entre las entidades involucradas en la ejecución del proceso es fundamental. Esta integración puede lograrse con una arquitectura orientada al servicio (SOA), lo cual sugiere identificar los servicios requeridos para dar soporte al proceso; por ejemplo, la optimización del recojo de ítems (figura 7).

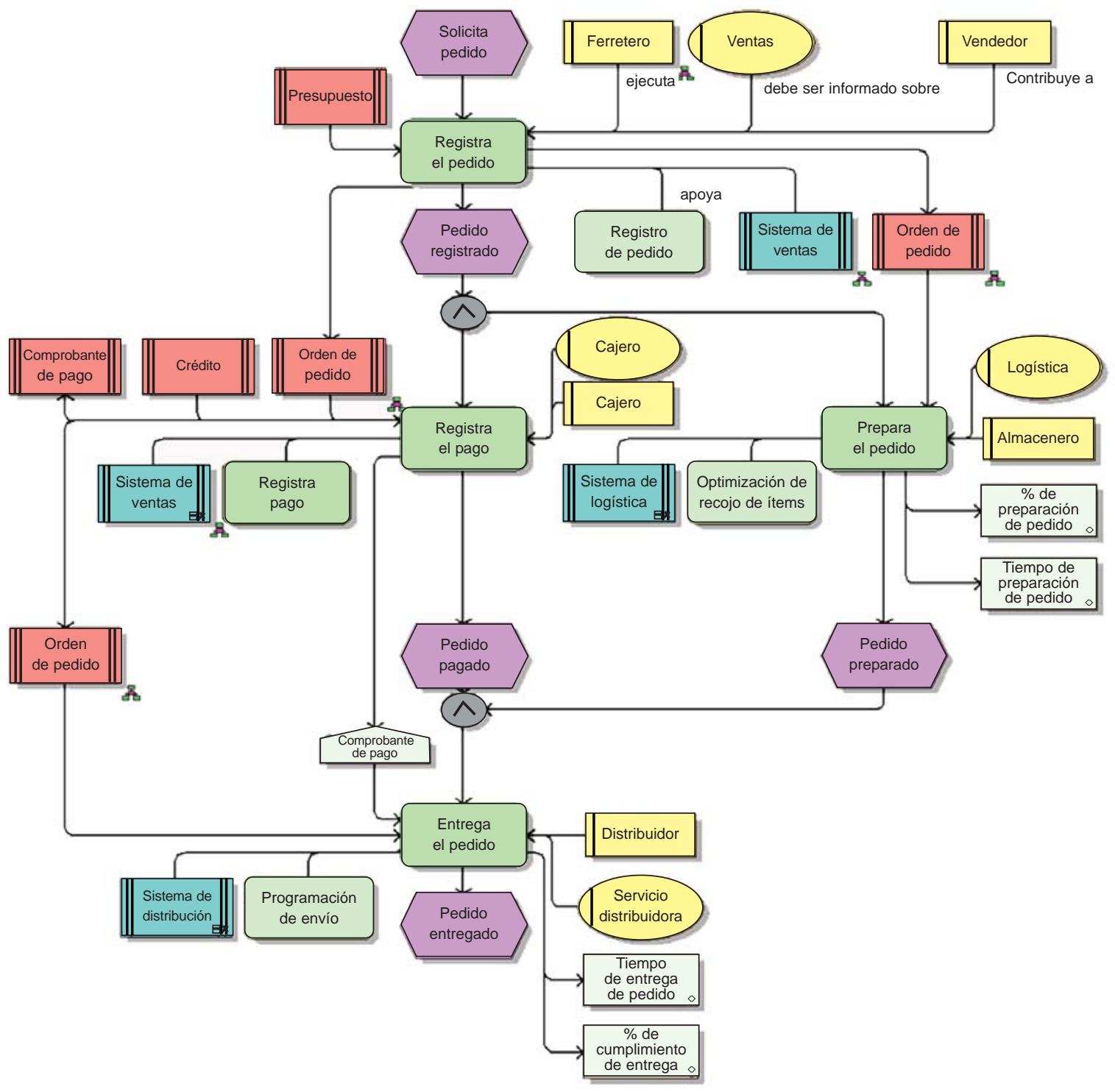

Figura 7. Orquestación y coreografía. 


\section{Arquitectura empresarial}

La integración empresarial requiere la realización de modelos con el propósito de describir diferentes aspectos de la organización, donde estos diagramas estén relacionados.

La necesidad más resaltante de la EA (por sus siglas en inglés) está dada por la velocidad de cambio del entorno empresarial. Donde la estrategia de agilidad organizacional es imperativa (IDS Scheer AG).

\section{Arquitectura de TI}

El desafío de los ejecutivos de TI es la adopción de una arquitectura de TI flexible, que se adapte a los requerimientos futuros del negocio. Los ejecutivos de TI también tienen el reto de crear una arquitectura modular, de ensamblaje, que pueda reconfigurarse para apoyar nuevas estrategias de negocio (Luftman).

\begin{tabular}{|c|c|c|c|}
\hline & Control de recursos & $\stackrel{\text { II }}{\text { Arquitectura de SI }}$ & Alineación estratégica \\
\hline $\begin{array}{l}\text { Perspectiva } \\
\text { administrativa }\end{array}$ & Funcional & Empresa & Red empresarial \\
\hline $\begin{array}{l}\text { Principal } \\
\text { producto de } \\
\text { planeación }\end{array}$ & Cartera de productos & $\begin{array}{l}\text { Arquitecturas } \\
\text { definidas }\end{array}$ & $\begin{array}{l}\text { Ajuste estratégico } \\
\text { externo/interno }\end{array}$ \\
\hline Beneficio & $\begin{array}{l}\text { Eficiencias mediante } \\
\text { automatización }\end{array}$ & $\begin{array}{l}\text { Flexibilidad de } \\
\text { productos mediante } \\
\text { arquitecturas }\end{array}$ & $\begin{array}{l}\text { Impulsado por el } \\
\text { mercado mediante } \\
\text { alineación dinámica }\end{array}$ \\
\hline $\begin{array}{l}\text { Administración } \\
\text { del valor }\end{array}$ & $\begin{array}{l}\text { Administración } \\
\text { de productos }\end{array}$ & $\begin{array}{l}\text { Despliegue } \\
\text { de políticas }\end{array}$ & $\begin{array}{l}\text { Estrategia } \\
\text { posibilitadora }\end{array}$ \\
\hline
\end{tabular}

Figura 8. El papel evolutivo de la planificación estratégica. 


\section{Conclusiones}

Una posición estratégica mediante la integración empresarial se logra:

- Mediante una posición estratégica singular.

- Alineando las estrategias, objetivos, iniciativas y procesos.

- Haciendo uso de los recursos de la red de valor.

- Mediante un modelo de operación que facilite la implementación de las iniciativas estratégicas.

- Mediante el SOA (véase el glosario), que facilita la integración de las aplicaciones para compartir datos.

- Mediante una arquitectura empresarial que agilice la respuesta a los cambios.

- Mediante una arquitectura de TI flexible, modular, que pueda reconfigurarse para apoyar las estrategias.

\section{Glosario}

Arquitectura empresarial. Es una abstracción de una empresa, sus elementos de varios tipos y sus relaciones (IDS Scheer).

La arquitectura empresarial clásica originada con la Industria de TI se divide en:

- Arquitectura del negocio, que define la estrategia, el gobierno, la estructura organizacional y los procesos de negocio.

- Arquitectura de aplicaciones, que provee el plan detallado de los sistemas de aplicación para ser desplegados, sus interacciones y sus relaciones con el proceso de negocio que soporta.

- Arquitectura de datos, que describe la organización lógica y física de los datos, así como la gestión de recursos.

- Arquitectura tecnológica, quel define el SW, el HW y la infraestructura de red pensada para soportar los sistemas de aplicación y bases de datos.

Coreografía. Definición de condición y secuencia de intercambio de mensajes entre o en los procesos de negocio (Lawler y Howell-Barber).

Integración horizontal. Es la integración lógica y física del proceso de negocio, desde la demanda del producto hasta su distribución. Consiste en el flujo del material y de documentos. Esta integración depende de la tecnología (Vernadat). 
Integración vertical. Viene a ser la integración de quienes toman las decisión y consiste en la integración de los sistemas de información para la toma de decisión. Es el flujo de decisión (Vernadat).

Modelo de operación. Describe cómo la organización desea prosperar y crecer (Ross, Weill y Robertson).

Orquestación. Definición de las reglas para el flujo de servicios en un proceso (Lawler y Howell-Barber).

Proceso de negocio. Es un conjunto estructurado y medible de actividades diseñadas con el fin de conseguir un resultado concreto para algún cliente o mercado específico (Thomas H. Davenport).

Servicio. Componente de aplicación desplegado en una red. Es descrito mediante Web Service y capaz de responder a los requerimientos de servicio (Lawler y Howell-Barber).

SOA. Arquitectura orientada a servicio, un enfoque para construir aplicaciones que implementa procesos o servicio de negocio usando un conjunto de componentes orquestados para entregar servicios con niveles definidos (Lawler y Howell-Barber; Hurwitz; Bloor; Baroudi y Kaufman).

\section{Bibliografía}

Davenport, Thomas H. Ecología de la información: ¿Por qué la tecnología no es suficiente para lograr el éxito en la era de la información? México: Oxford University Press, 1999.

Davis, Rob. ARIS design platform, advanced process modeling and administration. Londres: Springer, 2008.

Davis, Rob y Eric Brabander. AR/S design platform, Getting Started with BPM. Londres: Springer, 2007.

Harmon, Paul. Business process change: A manager's guide to improv ing, redesigning and automating process. San Francisco: Morgan Kaufmann Publishers, 2007.

IDS Scheer White Paper. Enterprise architecture and ARIS process platform. Marzo, 2005.

Kaplan, Robert S. y David P. Norton. Mapas estratégicos, convirtiendo los activos intangibles en resultados tangibles. 1. ${ }^{\text {a }}$ edición. Barcelona: Gestión 2000, 2004.

Keen, Peter y Mark MacDonald. Diferénciese con E-Process. Creación de valor para el cliente y riqueza para los negocios en la era de Internet. Bogotá: Osborne/McGraw-Hill, 2001. 
Lawler, James P. y H. Howell-Barber. Service-Oriented architecture, SOA strategy, methodology and technology. Nueva York: Auerbach Publications, Taylor y Francis Group, 2008.

Lawler, James P.; Howell-Barber, H.; Hurwitz, J.; Bloor, R.; Baroudi, C. y Marcia Kaufman. Service oriented architecture for dommies. Indianápolis: Wiley Published, 2007.

Luftman, J. N. La competencia en la era de la Información. México: Oxford University Press, 2001.

Peyret, H.; Leganza, G.; Hoekendijk, C.; King, O.; McCormack, M. y A. Carini. The Forrester Wave ${ }^{\mathrm{TM}}$ : Enterprise Herramientas de Arquitectura. Cambridge, MA: Forrester Research, Inc., 2007.

Ross, J. W.; Weill, P. y D. C. Robertson. Enterprise architecture as strategy. Cambridge, MA: Harvard Business School Press, 2006.

Rummler, G. A. y A. P. Brache. Improving performance, how to manage the white space on the organitation chart. 2. ${ }^{a}$ edición. San Francisco: Jossey-Bass Publushers, 1995.

Scheer, August-Wilhelm. Aris business process modeling. 2. ${ }^{a}$ edición. Berlín, 1999.

Scheer, A. W.; Kruppke, H.; Jost, H. \& H. Kindermann. Agility by ARIS business process modeling. Berlín: Springer, 2007.

Vernadat, F. B. Enterprise modeling and integration, principies and aplications. Londres: De Chapman \& Hall, 1996.

Woods, D. y T. Mattern. Enterprise SOA, design IT for business innovation. Nueva York: O'Reilly, 2006.

Weske, M. Business process management, concepts, languages, architectures. Berlín: Springer, 2007. 